\title{
Die Einleitung von gereinigtem Abwasser in Seen
}

\author{
Von H. BÜHRER und H. AMBÜHL
}

Eidg. Technische Hochschule Zürich, Institut für Gewässerschutz und Wassertechnologie, und Eidg. Anstalt für Wasserversorgung, Abwasserreinigung und Gewässerschutz (EAWAG)

Manuskript eingangen am 30. Mai 1975

\section{ABSTRACT \\ Discharge of Treated Wastewater in Lakes}

Treated wastewater discharged into a lake should be introduced below the trophogenic layer. Tracing the marked wastewater by echosounding revealed that the usual discharge method is unsuitable. The wastewater flows more or less to the depth of its own density, which is usually in the trophogenic layer. If there is no movement, that is, current in the water body, the wastewater accumulates at the pipe's mouth. A continuous current is therefore an indispensable prerequisite for a wastewater discharge site. It is only when wastewater is mixed with at least five parts of lake water that it does not rise more than two meters.

\section{INHALTSVERZEICHNIS}

1. Einleitung . . . . . . . . . . . . . . . . . . . . . . . . . . 348

2. Objekt, Methoden . . . . . . . . . . . . . . . . . . . . . . . . . . . . . 348

2.1 Berechnung der Wasserdichte. . . . . . . . . . . . . . . . . . . . . . . . . . 349

2.2 Berechnung der Schichtungsstabilität . . . . . . . . . . . . . . . . . . . . . 350

3. Messungen in situ . . . . . . . . . . . . . . . . . . . . . . . . . . . . . 351

3.5 Folgevungen aus den In-situ-Beobachtungen . . . . . . . . . . . . . . . . . . . . . 358

4. Die Frage der Einschichtung des eingeleiteten Abwassers . . . . . . . . . . 359

4.1 Die theoretische Einschichtungstiefe . . . . . . . . . . . . . . . . . . . . . . . 359

4.2 Das notwendige Mischungsverhältnis . . . . . . . . . . . . . . . . . . . . . . 361

5. Die Frage des Wegtransportes des eingeleiteten Abwassers . . . . . . . . . 366

6. Schlussbemerkungen. . . . . . . . . . . . . . . . . . . . . . . 366

7. Zusammenfassung, Summary, Résumé . . . . . . . . . . . . . . . . . . . . 367

Literaturverzeichnis . . . . . . . . . . . . . . . . . . . . . . 369 


\section{Einleitung}

Dass ausgerechnet die attraktivsten Anziehungspunkte unserer Landschaft und die mengenmässig bedeutendsten Trinkwasservorräte, nämlich die Seen, als Vorfluter für Siedlungsabwässer benützt werden, ist angesichts des steigenden Qualitätszerfalls vieler - wohl der meisten - Seen eine ebenso bedenkliche wie nicht $\mathrm{zu}$ übersehende Tatsache. Leider sind die Fälle, in denen eine andere Beseitigung der Abwässer technisch nicht möglich ist, zahlreich und wohl auch bis in fernere $\mathrm{Zu}$ kunft nur zum Teil zu ändern. Zwar sollte diese Art der Abwasserbeseitigung grundsätzlich eine Übergangslösung darstellen. Es ist aber - realistisch gesehen - damit zu rechnen, dass sie noch lange und in gewissen Fällen wohl für immer bestehen bleiben wird. Es ist deshalb ebenso realistisch, aus dieser unerfreulichen Situation das Beste zu machen und nach Lösungen zu suchen, welche diese Form der Entwässerung, die für jeden See prinzipiell schädlich ist, im Rahmen der verbleibenden Möglichkeiten wenigstens so wenig schädlich oder so optimal als möglich zu gestalten.

Nachdem Gächter [5] die Einleitung von gereinigtem Siedlungsabwasser in eine bestimmte seeindividuelle, auf Grund limnologischer Gegebenheiten zu berechnende Seetiefe begründet hat, soll nun versucht werden, praktische Richtlinien für die Realisierung dieser Forderungen zu erarbeiten.

\subsection{Bisherige Kenntnisse}

Die Frage, in welcher Weise sich Wasser anderer Dichte verhält, wenn es in einen stehenden Wasserkörper eingeleitet wird, wurde im Zusammenhang mit technischen Notwendigkeiten schon oft gestellt, bisher aber kaum jemals befriedigend beantwortet. Aus Modellversuchen [1] konnte gefolgert werden, dass sich Abwasser ungefähr auf der Höhe des Einleitungsortes einschichtet, wenn die Einleitung in den temperaturgeschichteten See turbulent erfolgt und dadurch eine Vermischung von Abwasser und Seewasser eintritt; die dazu erforderliche minimale Bewegungsenergie ist noch nicht bekannt.

Erfolgt der Abwasserausfluss bzw. die Einleitung laminar, so schichtet sich das Abwasser ohne merkliche Vermischung auf derjenigen Höhe ein, in der das Seewasser die gleiche Dichte aufweist. Ein derartiger Fall, bei welchem eine turbulente Vermischung bei der Ausmündung eines durch den Seespiegel rückgestauten offenen Einleitungskanals fehlte, ist am Pfäffikersee nachgewiesen worden [3].

Die Notwendigkeit, eine verständlicherweise abwartende, für die betroffenen Seen indessen mit Sicherheit schädliche Politik durch realisierbare Richtlinien möglichst rasch zu verbessern, bewog uns, das Problem der Einleitungstechnik experimentell anzugreifen und entsprechende Verhältnisse an einem bestehenden Objekt in einem See im Maßstab 1:1 zu prüfen.

\section{Objekt, Methoden}

Für die Messungen wurde die Kläranlage Hergiswil am Vierwaldstättersee gewählt (vgl. Abb. 5). Das Abwasser wird am Steilufer in $-11 \mathrm{~m}, 30 \mathrm{~m}$ vom Ufer 
entfernt, durch ein etwa $2 \mathrm{~m}$ über Grund aufgebocktes Rohr in den See geleitet. Die Stelle dieser Einleitung ist dank dem nahen, steilen Ufer leicht zu orten.

Auf Grund von Vorversuchen wurde das Abwasser in der nahe gelegenen Kläranlage mit Al-Pulver markiert (Alu-Pulver 40 p der Firma Doral, CH-1963 Vétrox). Dieses für die Herstellung von "Silberbronze» verwendete Material besteht aus AlPlättchen von etwa $40 \mu$ Durchmesser und rund 0,2 $\mu$ Dicke; sie sind beidseits mit Paraffin beschichtet. Diese Plättchen stellen sich im Wasser, dem zur Aufschwemmung ein Detergent beigegeben werden muss, zum grossen Teil parallel und bilden auf diese Weise eine scheinbare Fläche, welche die Wellenlänge des zur Objektortung verwendeten "Atlas»-Echographen ( $3 \mathrm{~cm}$ Ultraschall, etwa $50 \mathrm{kHz}$ ) gut reflektiert, obschon die Partikelgrösse allein diese Reflexe theoretisch nicht hervorrufen kann. Die Dosierung wurde im Mess- und Auslaufschacht der Kläranlage mit Hilfe eines Rührwerks (zum Aufrechterhalten der Suspension) und einer Dosierpumpe vorgenommen; es wurde bei einem Zusatz von rund $1 \mathrm{~g}$ Al-Pulver pro $1 \mathrm{~m}^{3}$ Abwasser jeweils rund 45 Minuten dosiert.

Die Aufzeichnung der markierten Abwasserfahne ist, wie die Echogramme in Kapitel 3 zeigen, klar; schwieriger ist die genaue horizontale Ortung der Fahne. Da der im Boot fest eingebaute Sender-Empfänger des Echographen nur vertikal unter dem Schiff befindliche Objekte registrieren kann, ist es beispielsweise fast unmöglich, den longitudinalen Verlauf der Fahne auf längere Strecke zu verfolgen, indem die einmal verlorene markierte Fahne auf der gleichen Messfahrt normalerweise nicht mehr zu finden ist.

Diese Nachteile konnten mit Hilfe eines beweglichen fernsteuerbaren, ausser Bord montierten Sender-Empfängers vermieden werden, allerdings unter Inkaufnahme einer Beschränkung des Messbereichs, indem bei dieser Anordnung anfänglich nur Tiefen von $>7 \mathrm{~m}$ erfasst werden konnten (bei normaler Ausrüstung $>4-5 \mathrm{~m}$ ). Der Sender-Empfänger wurde zu diesem Zweck auf einem Kreuzgelenk allseits beweglich, aber verdrehungssicher gelagert und durch einen Steuerknüppel über 4 kreuzweise befestigte Bowdenzüge bewegt.

\subsection{Berechnung der Wasserdichte}

Die analytische Bestimmung der Dichte einer verdünnten Lösung ist mit den üblichen Mitteln für die hier vorliegende Aufgabe zu wenig genau. Aus diesem Grund wurde die Dichte des geschichteten Seewassers nicht direkt gemessen, sondern auf Grund seiner Temperatur und der elektrischen Leitfähigkeit berechnet. Dazu wurden folgende Quellen benützt:

- Dichten von destilliertem Wasser: Handbook of Chemistry and Physics, 45. Auflage;

- Umrechnung der aktuellen Leitfähigkeit bei $20^{\circ} \mathrm{C}$ : Tabelle EAWAG (Basis: Wasser aus dem Bodensee-Untersee [2]).

- Annahme, dass 1. Siemens $/ \mathrm{cm}$ bei $20^{\circ} \mathrm{C} 0,8 \mathrm{mg} / \mathrm{cm}^{3}$ gelöster Substanz entspricht und dass $1 \mathrm{mg} / \mathrm{l}$ gelöste Substanz einer Dichtezunahme von $0,0008 \mathrm{~g} / \mathrm{cm}^{3}$ entspricht. Dieser Ansatz stimmt annäherungsweise für $\mathrm{Ca}\left(\mathrm{HCO}_{3}\right)_{2}$.

Diese Beziehungen wurden analytisch folgendermassen realisiert: Temperaturkorrektur der Leitfähigkeit: 


$$
x_{20^{\circ}}=\varkappa \cdot\left(-0,00001222651 \cdot \mathrm{T}^{3}+0,00114842 \cdot \mathrm{T}^{-2}-0,0541369 \cdot \mathrm{T}+1,72118\right) ;
$$

Temperaturkorrektur der Dichte:

$$
\begin{gathered}
\mathrm{d}_{\mathrm{H}_{2} \mathrm{O}}=\left(0,059385 \cdot \mathrm{T}^{3}-8,56272 \cdot \mathrm{T}^{2}+65,4891 \cdot \mathrm{T}\right) \cdot 10^{-6}+0,99984298 \\
\quad\left(\text { gilt zwischen } 0^{\circ} \mathrm{C} \text { und } 24^{\circ} \mathrm{C} \text { mit } \pm 0,000001\right) ;
\end{gathered}
$$

Berechnung der Dichte des Seewassers:

$$
\mathrm{d}_{\text {Seewasser }}=\mathrm{d}_{\mathrm{H}_{2} \mathrm{O}}+0,64 \cdot 10^{-6} \cdot \varkappa_{20^{\circ}} \text {. }
$$

Symbole: $\mathrm{d}=$ Dichte in $\mathrm{g} / \mathrm{cm}^{-3}$

$\mathrm{d}_{\mathrm{H}_{8} \mathrm{O}}=$ Dichte von destilliertem Wasser in $\mathrm{g} / \mathrm{cm}^{-3}$

$\mathrm{T}=$ Temperatur in Grad Celsius

$\varkappa \quad=$ Leitfähigkeit in $\mu \mathrm{S} / \mathrm{cm}^{-1}$

\subsection{Berechnung der Schichtungsstabilität}

Die Stabilität der thermischen oder Dichteschichtung ist für alle Erscheinungen im Wasserkörper, an denen die Schichtung als Phänomen oder physikalischer Zustand Anteil hat ( \pm alle chemischen und biologischen Prozesse im Epilimnion) eine ausserordentlich wichtige Grösse. Sie ist für das erstaunlich dauerhafte Gefüge des sommerlichen stratifizierten Wasserkörpers verantwortlich. Sie wurde nach folgendem Verfahren berechnet.

Als Vereinfachung wurde angenommen, dass die Dichte mit der Tiefe linear zunehme. Dann wurde die Differenz zwischen dem wirklichen (bzw. berechneten) und dem homothermen (= für die gleichmässig temperierte Schicht gültigen) Schwerpunkt der Schicht $\left(t_{2}-t_{1}\right)$ mit dem berechneten Gewicht multipliziert und als Schichtungsstabilität bezeichnet.

$$
\begin{aligned}
& \text { Dichte to } \mathrm{m}^{-3} \\
& \begin{array}{|l|l}
\hline t_{1} & \\
t_{h}-t_{b} & \\
t_{2} & \\
& \\
\end{array} \\
& \text { Tiefe } \\
& \text { m } \\
& \mathrm{d}=\mathrm{f}(\mathrm{t}) \quad \mathrm{G}_{\mathrm{b}}=\left(\mathrm{d}_{1}+\mathrm{d}_{2}\right)\left(\mathrm{t}_{2}-\mathrm{t}_{1}\right) / 2 \\
& t_{b}=\int_{t_{1}}^{t_{2}} t \cdot f(t) d t / G_{b} \\
& t_{b}=\frac{2 / 3\left(t^{2}+t_{1} t_{2}+t^{2}\right)\left(d_{2}-d_{1}\right)+d_{1}\left(t_{2}^{2}-t_{1}^{2}\right)-t_{1}\left(t_{2}-t_{1}\right)\left(d_{2}-d_{1}\right)}{\left(d_{1}+d_{2}\right)\left(t_{2}-t_{1}\right)} \\
& \text { Stabilität }=\frac{G_{b}\left(t_{b}-t_{b}\right)}{\left(t_{2}-t_{1}\right)^{3}} \quad t_{h}=\left(t_{2}-t_{1}\right) / 2
\end{aligned}
$$


Symbole: $\mathrm{d}=$ Dichte

$\mathrm{G}_{\mathrm{b}}=$ Berechnetes Gewicht

$\mathrm{t}=$ Tiefe

$t_{b}=$ Tiefe des berechneten Schwerpunkts

$\mathrm{t}_{\mathrm{h}}=$ Tiefe des Schwerpunkts bei Homothermie

In den nachstehend beschriebenen Messungen wurden die in Tabelle 1 zusammengestellten Stabilitätswerte ermittelt.

Tabelle 1. Ubbersicht über die Stabilitäten im Vierwaldstättersee.

Table 1. Stabilities survey in the Lake of Lucerne.

\begin{tabular}{llll}
\hline Datum & $\begin{array}{l}\text { Maximum } \\
\mathrm{kp} / \mathrm{m}^{2}\end{array}$ & $\begin{array}{l}\text { Minimum } \\
\mathrm{kp} / \mathrm{m}^{2}\end{array}$ & $\begin{array}{l}\text { An der Einschichtungsgrenze } \\
\mathrm{kp} / \mathrm{m}^{2}\end{array}$ \\
\hline 29.8 .1973 & 0,0751 & 0,00098 & 0,017 \\
26.9 .1973 & 0,0220 & $-0,0028$ & 0,0035 \\
16.7 .1974 & 0,0714 & $-0,00189$ & 0,010 \\
31.7 .1974 & 0,0474 & 0,00277 & 0,014 \\
\hline
\end{tabular}

\section{Messungen in situ}

Wie erwähnt, erwies sich die Navigation als schwieriger als die eigentliche Echolotmessung. Eine Einmessung des relativ rasch fahrenden Meßschiffes vom Land aus war technisch nicht möglich. Dagegen konnten die Stützen der Autobahn HergiswilLopper als Nord-Süd-Fluchtlinien verwendet werden. West-Ost-Fluchtlinien, die eine Orientierung entlang der Abwasserleitung ermöglichten, wurden durch Jalons am Ufer abgesteckt.

Vor den Abwassermessungen wurde jeweils die Temperatur- und Leitfähigkeitsschichtung im See sowie Menge, Temperatur und Leitfähigkeit des Abwassers gemessen. In der zu erwartenden Einschichtungstiefe wurden sodann mit Hilfe von Driftkörpern die Strömungsverhältnisse ermittelt, im Versuch vom 26. September 1973 durch Einmessung mit Theodoliten unter Mitarbeit der VAW, in den übrigen Versuchen durch Einpeilung und Schätzung.

\subsection{Versuch $N r .1$ vom 29. August 1973}

Die Messdaten für Temperatur und die berechneten Werte für Leitfähigkeit bei $20^{\circ} \mathrm{C}$, Dichte und Stabilität sind in Tabelle 2 zusammengestellt.

\section{Einschichtungstiefe}

Die in Abb. 1 dargestellten Ausschnitte aus den Echolotregistrierungen zeigen, dass das Abwasser unmittelbar vom Austritt aus dem Rohr an in einer unregelmässigen Bewegung aufsteigt und sich mit Obergrenze etwas höher als $5 \mathrm{~m}$, untere Grenze bei 7,5 m, einschichtet. Diese Höhenlage ist stabil; bei der Längsverteilung, die etwa $90 \mathrm{~m}$ weit verfolgt werden konnte, hält sie sich konstant (Abb.2). 
Tabelle 2. Seeparameter und Stabilität während des Versuchs 1 am 29. August 1973.

Table 2. Parameter and stability of the lake during trial 1 (29 August 1973).

\begin{tabular}{llllr}
\hline $\begin{array}{l}\text { Tiefe } \\
\mathrm{m}\end{array}$ & $\begin{array}{l}\text { Temperatur } \\
{ }^{\circ} \mathrm{C}\end{array}$ & $\begin{array}{l}\text { Leitfähigkeit } \\
\mu \mathrm{S} 20^{\circ} \mathrm{C}\end{array}$ & $\begin{array}{l}\text { Dichte } \\
\mathrm{g} / \mathrm{cm}^{3}\end{array}$ & $\begin{array}{r}\text { Stabilität } \\
\mathrm{p} / \mathrm{m}^{2}\end{array}$ \\
\hline 0 & 23,25 & 163,6 & 0,997588 & 1,9606 \\
1 & 23,15 & 163,5 & 0,997611 & 1,9531 \\
2 & 23,05 & 163,4 & 0,997634 & 43,9827 \\
3 & 20,7 & 166,3 & 0,998162 & 75,1611 \\
4 & 15,9 & 166,3 & 0,999064 & 18,1747 \\
$\mathbf{5}$ & 14,5 & 171,1 & 0,999282 & 1,1516 \\
6 & 13,55 & 172,9 & 0,999416 & 2,8782 \\
7 & 13,3 & 175,2 & 0,999451 & 5,5879 \\
8 & 12,75 & 170,5 & 0,999518 & 3,6322 \\
9 & 12,4 & 172,1 & 0,999561 & 3,5037 \\
10 & 12,05 & 173,6 & 0,999603 & 0,9773 \\
11 & 11,95 & 174,1 & 0,999615 & 1,3809 \\
12 & 11,8 & 173,6 & 0,999632 & 0,9179 \\
13 & 11,7 & 173,4 & 0,999643 & 1,4384 \\
14 & 11,55 & 174,7 & 0,999660 & 1,4475 \\
15 & $\mathbf{1 1 , 4}$ & 176,6 & 0,999677 & 1,3576 \\
16 & 11,25 & 177,3 & 0,999694 & $(351 / \mathrm{s})$ \\
Abwasser & 17,25 & 543,8 & 0,999077 & \\
\hline
\end{tabular}

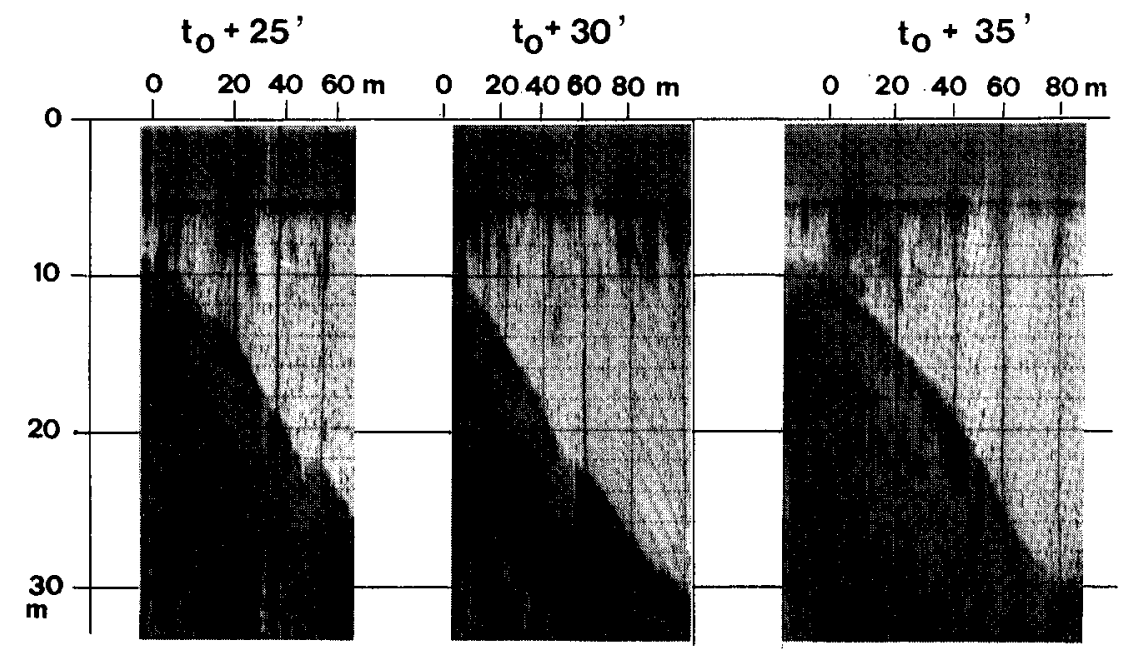

Abb. 1. Echogramme. Längsschnitte durch die Abwasserfahne am 29. August 1973. $t_{0}=$ Zeitpunkt des Starts der Aluminiumzudosierung (obere $5 \mathrm{~m}$ werden durch. Störsignale ausgelöst). Lücken im Echo des Abwassers sind durch das Schwenken des Sendeempfängers bedingt und daher zu ergänzen.

Fig. 1. Longitudinal section of the wastewater plume on 29 August 1973. $t_{0}=$ time of starting dosage of aluminium (uppermost $5 \mathrm{~m}$ extincted by interferences); blanks in the echogramme are caused by turning the transmitter-receiver and should therefore be completed. 


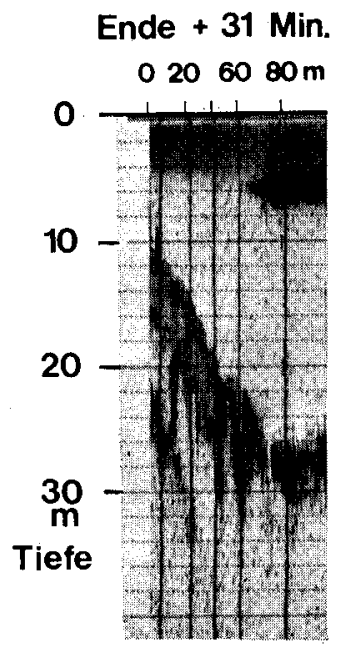

Abb. 2. Längsschnitt durch die Abwasserfahne 31 Minuten nach Ende der Aluminiumdosierung.

Fig. 2. Longitudinal section of the plume 31 minutes after stopping dosage of aluminium.

Abb. 3 zeigt die aus den Echogrammen umgezeichneten und entzerrten Einzeldiagramme der entsprechenden Messfahrten.
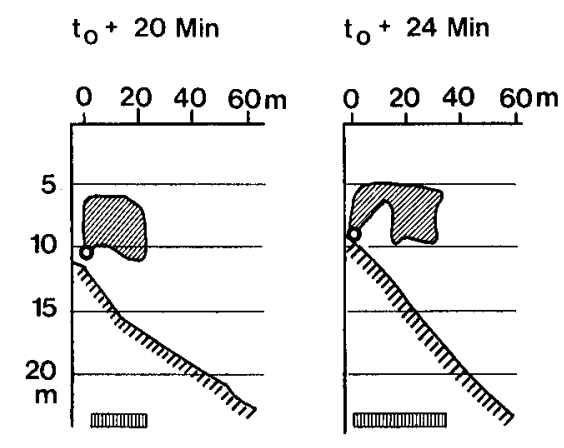

$$
t_{0}+30 \mathrm{Min}
$$$$
t_{0}+35 \operatorname{Min}
$$
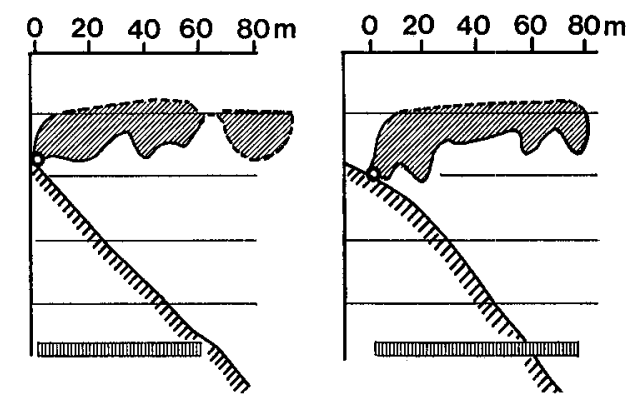

Abb. 3. Rekonstruktion des zeitlichen Verlaufs der Abwasserfahne vom 28. August 1973. Fig. 3. Reconstruction of the chronological sequence of the wastewater plume from 28 August 1973.

Nach Abb. 4 (graphische Darstellung der Messdaten aus Tab. 2) würde die theoretische Einschichtungstiefe, der Dichteverteilung entsprechend, zwischen 4 und $5 \mathrm{~m}$ liegen. Der grosse Dichtegradient bzw. die hohe Stabilität in der Schicht über 4,5 m machten ein weiteres Aufsteigen, obschon dies aus den Echogrammen nicht ersichtlich ist, wahrscheinlich unmöglich. Das Abwasser wird relativ rasch und kompakt durch eine entsprechende Strömung weggeführt, ohne sich im weiteren Verlauf wesentlich mit dem Seewasser zu vermischen. 


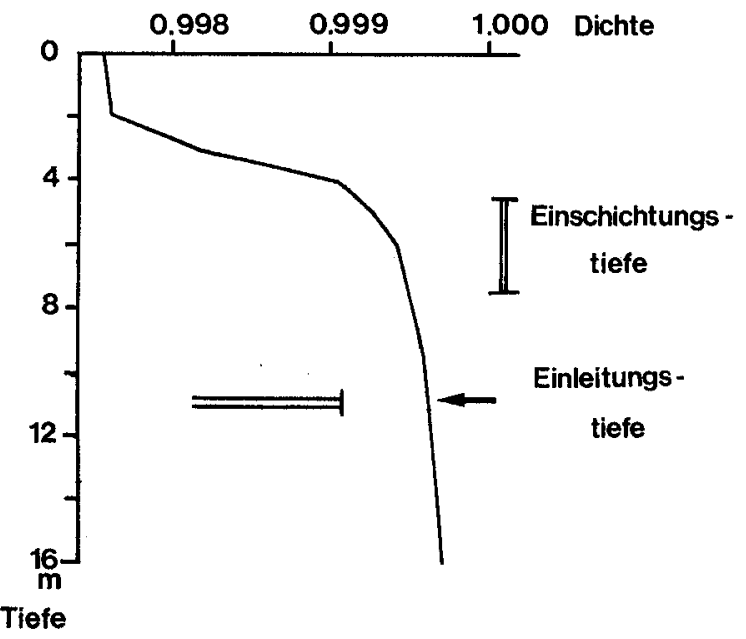

Abb. 4. Dichteschichtung der Hergiswiler Bucht am 29. August 1973.

Fig. 4. Densities in Hergiswil Bay on 29 August 1973.

\subsection{Versuch Nr. 2 vom 26. September 1973}

Die Messdaten dieser Beobachtung sind in Tabelle 3 zusammengestellt.

Tabelle 3. Seeparameter und Stabilität während des Versuchs 2 am 26. September 1973. Table 3. Parameter and stability of the lake during trial 2 (26 September 1973).

\begin{tabular}{llllc}
\hline $\begin{array}{l}\text { Tiefe } \\
\mathrm{m}\end{array}$ & $\begin{array}{l}\text { Temperatur } \\
{ }^{\circ} \mathrm{C}\end{array}$ & $\begin{array}{l}\text { Leitfähigkeit } \\
\mu \mathrm{S} 20^{\circ} \mathrm{C}\end{array}$ & $\begin{array}{l}\text { Dichte } \\
\mathrm{g} / \mathrm{cm}^{\mathbf{3}}\end{array}$ & $\begin{array}{c}\text { Stabilität } \\
\mathrm{p} / \mathrm{m}^{2}\end{array}$ \\
\hline 0 & 15,9 & 163,0 & 0,999062 & $-2,787$ \\
1 & 16,1 & 161,7 & 0,999029 & $-0,666$ \\
2 & 16,15 & 162,2 & 0,999021 & 0,695 \\
3 & 16,1 & 162,2 & 0,999029 & 0,0585 \\
4 & 16,1 & 163,3 & 0,999030 & 0,0000 \\
5 & 16,1 & 163,3 & 0,999030 & 3,471 \\
6 & 15,85 & 164,9 & 0,999071 & 6,854 \\
7 & 15,35 & 169,7 & 0,999154 & 22,022 \\
8 & 13,6 & 186,1 & 0,999418 & 5,696 \\
9 & 13,1 & 190,2 & 0,999486 & 4,006 \\
10 & 12,6 & 167,6 & 0,999534 & 3,608 \\
11 & 12,25 & 169,7 & 0,999578 & 0,533 \\
12 & 12,2 & $\mathbf{1 7 0 , 5}$ & 0,999584 & 3,461 \\
13 & 11,85 & 172,7 & 0,999625 & 3,759 \\
14 & 11,45 & 174,5 & 0,999671 & 2,229 \\
15 & 11,2 & 175,1 & 0,999697 & $(501 / \mathrm{s})$ \\
Abwasser & 14,2 & 584,8 & 0,999590 & \\
\hline
\end{tabular}

Während die erste Messung noch in der Stagnationsphase des Sees gemacht wurde, fiel Nr. 2 in die beginnende Zirkulationsphase, mit Mischung des Wasserkörpers bis auf $5 \mathrm{~m}$ und vorauslaufender interner unvollständiger Mischung auf 7,5 m, mit einem markanten Stabilitätsanstieg in dieser Tiefe. 


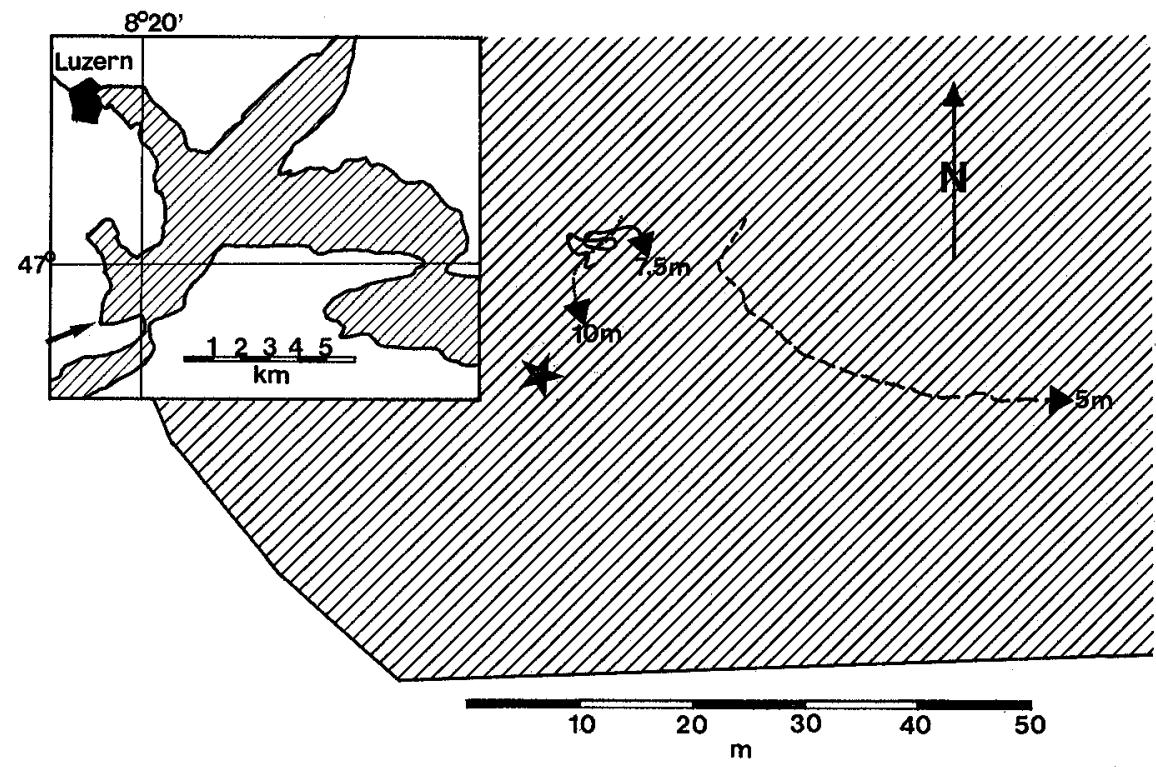

Abb. 5. Wege der Driftkörper während 35 Minuten in verschiedenen Tiefen am 26. September 1973. (Messungen der Versuchsanstalt für Wasserbau, Hydrologie und Glaziologie ETHZ).

Fig. 5. Driftbody paths observed for 35 minutes in different depths on 26 September 1973 (measurements of VAW).

Während der Messzeit war die Hergiswiler Bucht, wie die Horizontalmessungen der VAW ergeben, nahezu strömungsfrei (Abb. 5).

Die Echolotmessungen ergeben, dass das eingeleitete Wasser nicht weggeführt wurde, sondern dass es sich in der näheren Umgebung der Einleitungsstelle in Form einer Blase ansammelte. Das während der 40minütigen Versuchsdauer eingeleitete markierte Abwasser umfasste bei einem mittleren Auslauf von $50 \mathrm{l} / \mathrm{s}$ rund $700 \mathrm{~m}^{3}$. Die Echogramme, die während dieser Zeit aufgezeichnet werden konnten, erlaubten eine Rekonstruktion dieser "Abwasserblase» (Abb. 6) mit einem Volumen von etwa $650 \mathrm{~m}^{3}$. Dies bedeutet, dass praktisch die gesamte Abwassermenge im See wieder gefunden wurde und dass die Vermischung mit dem Umgebungswasser unter den örtlichen Verhältnissen, die von jenen anderer ARAs kaum wesentlich abweichen, ausserordentlich gering ist, trotz einer Ausströmungsgeschwindigkeit des Abwassers von rund $1,6 \mathrm{~m} / \mathrm{s}$.

Die Messungen wurden während ausgesprochener Sommerstagnation durchgeführt mit einem (temperaturbedingten) Stabilitätsmaximum in einer Tiefe zwischen 4 und $5 \mathrm{~m}$. Die Aufzeichnungen im Echographen (Abb. 7) erfassen nur die frisch aus dem Rohr ausgetretene Fahne; infolge eines zu spät entdeckten Defektes an der Tracerzufuhr wurde der Aluzusatz wesentlich verdünnter dosiert als in den übrigen Versuchen, weshalb die aufgezeichneten Echos weniger auf Reflexe am Tracermaterial als auf den grossen Dichtegradienten zwischen der noch nicht eingeschich- 
teten Fahne und dem umgebenden Seewasser zurückzuführen ist. (Derartige Dichtesprünge und auch turbulente Bewegungen reflektieren Ultraschall und werden vom Echolot erfasst.)
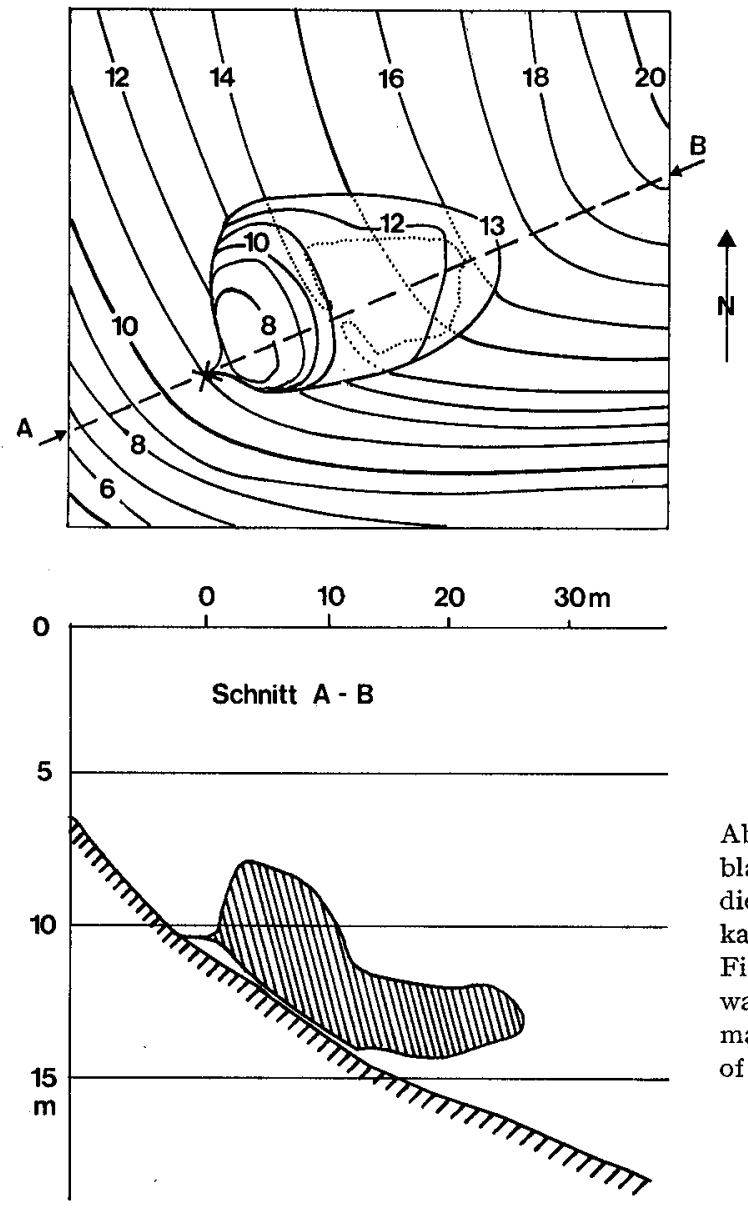

Abb. 6. Rekonstruktion der Abwasserblase von Versuch 2. Der Stern bedeutet die Abwassereinleitungsstelle (Tiefenkarte, Äquidistanz der Tiefenlinien $1 \mathrm{~m}$ ). Fig. 6. Reconstruction of the wastewater cloud of trial 2. The asterisk marks the point of discharge (interval of lines of depth $1 \mathrm{~m}$ ).

\subsection{Versuch Nr. 3 vom 16. Juli 1974 (Messdaten siehe Tab. 4)}

Die Dichte des Abwassers wiederholte sich im See gemäss der berechneten vertikalen Dichteverteilung in 4-5 m. Das Abwasser zeichnete sich, soweit aus den Echogrammen ersichtlich, in 6,5-8,5 m ein, wobei die Einschichtung nach 7,5 $\mathrm{m}$ Horizontaldistanz schon einigermassen und nach $20 \mathrm{~m}$ vollständig abgeschlossen war. Der Wegtransport der Abwasserfahne erfolgte durch eine entsprechende Seeströmung. 


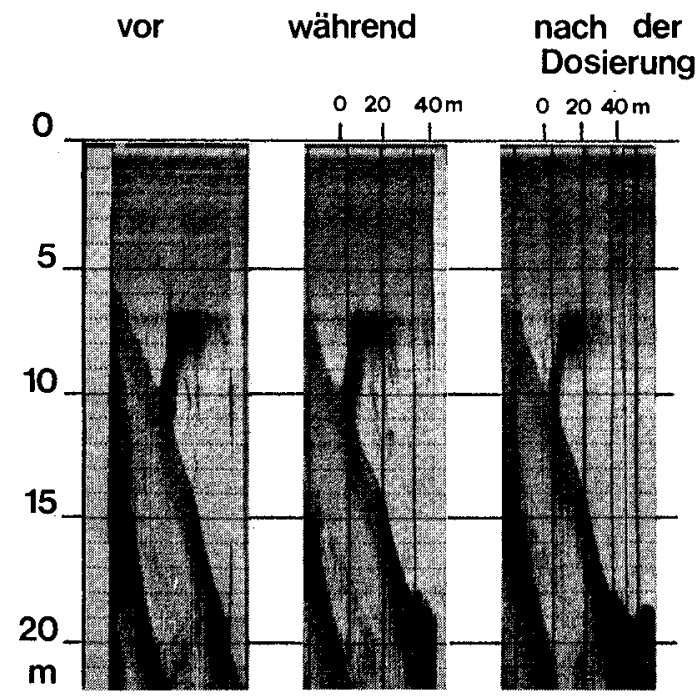

Abb. 7. Echogramme des schwach markierten Abwassers am 16. Juli 1974 (Versuch 3). Fig. 7. Echogrammes of only slightly marked wastewater on 16 July 1974 (trial 3).

Tiefe

Tabelle 4. Seeparameter und Stabilität während des Versuchs 3 am 16. Juli 1973. Table 4. Parameter and stability of the lake during trial 3 (16 July 1973).

\begin{tabular}{llllr}
\hline $\begin{array}{l}\text { Tiefe } \\
\mathrm{m}\end{array}$ & $\begin{array}{l}\text { Temperatur } \\
{ }^{\circ} \mathrm{C}\end{array}$ & $\begin{array}{l}\text { Leitfähigkeit } \\
\mu \mathrm{S} 20^{\circ} \mathrm{C}\end{array}$ & $\begin{array}{l}\text { Dichte } \\
\mathrm{g} / \mathrm{cm}^{3}\end{array}$ & $\begin{array}{c}\text { Stabilität } \\
\mathrm{p} / \mathrm{m}^{2}\end{array}$ \\
\hline 0 & 21,2 & 186,8 & 0,998068 & $-1,891$ \\
1 & 21,3 & 185,4 & 0,998046 & 3,794 \\
2 & 21,1 & 188,7 & 0,998091 & 8,936 \\
3 & 20,6 & 188,9 & 0,998198 & 22,023 \\
4 & 19,3 & 185,0 & 0,998463 & 71,396 \\
5 & 14,25 & 172,1 & 0,999319 & 6,953 \\
6 & 13,65 & 172,4 & 0,999403 & 3,239 \\
7 & 13,35 & 170,8 & 0,999442 & 6,753 \\
8 & 12,75 & 177,7 & 0,999523 & 3,888 \\
9 & 12,35 & 174,7 & 0,999569 & 1,9112 \\
10 & 12,15 & 173,8 & 0,999592 & 1,673 \\
11 & 12,0 & 178,1 & 0,999612 & 1,811 \\
12 & 11,8 & 176,6 & 0,999634 & 0,977 \\
13 & 11,7 & 177,7 & 0,999646 & $(401 / \mathrm{s})$ \\
Abwasser & 17,5 & 589,3 & 0,999062 & \\
\hline
\end{tabular}

\subsection{Versuch Nr. 4 vom 31.Juli 1974 (Messdaten siehe Tab. 5)}

Entsprechend der hohen Abwassertemperatur war bei dieser Messung zu erwarten, dass sich das Abwasser ziemlich hoch einschichten würde, nämlich in rund $3 \mathrm{~m}$. Da die Echolotaufzeichnung nur von $5 \mathrm{~m}$ an einwandfrei ist, kann die endgültige Einschichtungstiefe nicht genau angegeben werden; gemäss Abb. 8 (Querschnitte 
Tabelle 5. Seeparameter und Stabilität während des Versuchs 4 am 31. Juli 1974.

Table 5. Parameter and stability of the lake during trial 4 (31 July 1974).

\begin{tabular}{llllr}
\hline $\begin{array}{l}\text { Tiefe } \\
\mathrm{m}\end{array}$ & $\begin{array}{l}\text { Temperatur } \\
{ }^{\circ} \mathrm{C}\end{array}$ & $\begin{array}{l}\text { Leitfähigkeit } \\
\mu \mathrm{S} 20^{\circ} \mathrm{C}\end{array}$ & $\begin{array}{l}\text { Dichte } \\
\mathrm{g} / \mathrm{cm}^{\mathbf{3}}\end{array}$ & $\begin{array}{l}\text { Stabilität } \\
\mathrm{p} / \mathrm{m}^{2}\end{array}$ \\
\hline 0 & 21,25 & 172,1 & 0,998047 & 16,211 \\
1 & 20,35 & 175,6 & 0,998242 & 47,374 \\
2 & 17,40 & 168,9 & 0,998811 & 16,959 \\
3 & 16,2 & 164,6 & 0,999014 & 13,326 \\
4 & 15,2 & 165,2 & 0,999174 & 13,166 \\
5 & 14,15 & 170,3 & 0,999332 & 7,739 \\
6 & 13,5 & 176,0 & 0,999425 & 6,368 \\
7 & 12,9 & 174,0 & 0,999501 & 3,753 \\
8 & 12,55 & 176,8 & 0,999546 & 5,016 \\
9 & 12,05 & 178,5 & 0,999606 & 2,772 \\
10 & 11,75 & 177,5 & 0,999640 & $(301 / \mathrm{s})$ \\
Abwasser & 17,9 & 519,7 & 0,998944 & \\
\hline
\end{tabular}

durch Fahne) und Rekonstruktion eines Längsschnittes (Abb. 9) kann immerhin angegeben werden, dass die untere Begrenzung der Fahne höher als $5 \mathrm{~m}$ lag, wobei der Aufstieg verhältnismässig langsam erfolgte (innerhalb $25 \mathrm{~m}$ Horizontaldistanz).

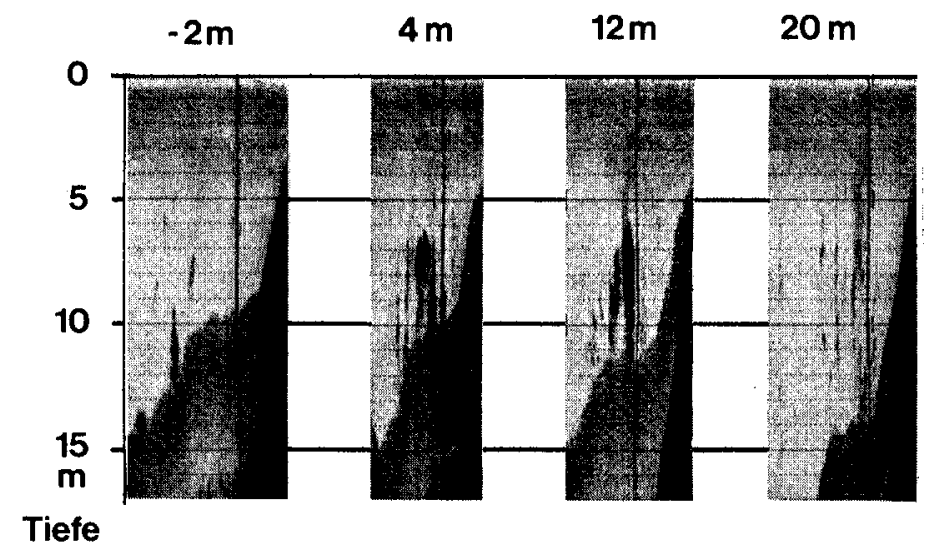

Abb. 8. Echogramme der Abwasserfahne am 31. Juli 1974, Versuch 4 (Querschnitte hinter und vor der Einleitungsstelle).

Fig. 8. Echogramme of the wastewater plume on 31 Juli 1974. Cross sections in front of and behind the point of discharge.

\subsection{Folgerungen aus den In-situ-Beobachtungen}

Während unserer Beobachtungen herrschten am Auslauf der Kläranlage Hergiswil Reynoldszahlen im Rohr von $1,3 \cdot 10^{5}$ bis $1,9 \cdot 10^{5}$ (bzw. Rohrgeschwindigkeiten von 0,45 bis $0,75 \mathrm{~m} / \mathrm{s}$ ). Die Rohrströmung war somit immer turbulent; eine laminare Fortsetzung im See ist ausgeschlossen. Ferner ergeben die In-situ-Beobachtungen 


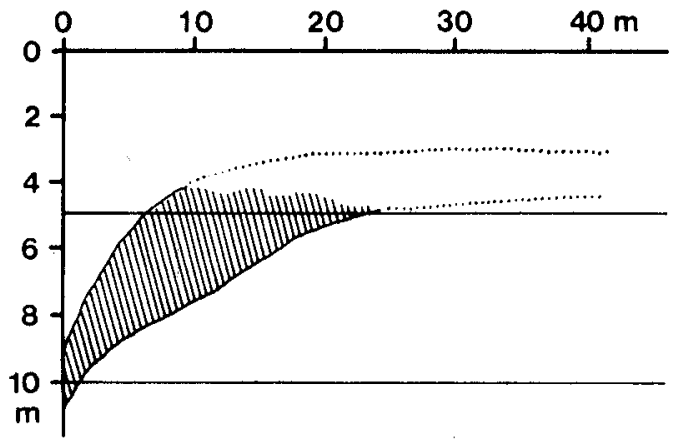

Abb. 9. Rekonstruktion der Abwasserfahne am 31. Juli 1974 um 14 Uhr. Die Fahne steigt höher als $5 \mathrm{~m}$ und lässt sich dann mit unseren Mitteln nicht mehr verfolgen.

Fig. 9. Scheme of wastewater on 31 July 1974 at 2 p.m. The plume rises higher than $5 \mathrm{~m}$, so that it can no longer be traced by our method.

eindeutig, dass das eingeleitete Abwasser mit dem umgebenden Seewasser der Einleitungstiefe nur teilweise, jedenfalls völlig ungenügend, vermischt wird und sich erstaunlich rasch annähernd in der Tiefe der gleichen Dichte einschichtet. Dies bedeutet, dass frïhere theoretische Vorstellungen [3] zutreffen, und dass der heute in der Praxis übliche Modus der Einleitung überprüft werden sollte.

\section{Die Frage der Einschichtung des eingeleiteten Abwassers}

\subsection{Die theoretische Einschichtungstiefe}

Die vier Experimente machen klar, dass das eingeleitete Abwasser nicht in der Einleitungstiefe verbleibt, sondern dass es sich in jener Tiefe einschichtet, welche die gleiche Dichte hat. Diese Tatsache ist von eminenter praktischer Bedeutung. Soll nämlich das Abwasser in einer andern als dieser "natürlichen» Tiefe verbleiben, so ist dies nur durch Anpassung seiner Dichte möglich, was - realistisch gesehen einzig durch Vermischung mit Seewasser aus diesem Horizont zu erzielen ist. Dabei stellt sich die Frage nach dem erforderlichen Mischungsverhältnis.

Diese Frage wirft etliche Detailprobleme auf. Im folgenden versuchen wir, diese sowie das Gesamtproblem anhand der während der Versuche festgestellten Dichtestratifikation sowie von Temperatur- und Leitfähigkeitsmessungen aus dem Bodensee zu optimieren 1 ). Für die folgenden Mischungsrechnungen treffen wir die vereinfachende Annahme, dass

a) kein Wärmetransport (unabhängig vom Massentransport) stattfindet,

b) die Leitfähigkeit bei $20^{\circ} \mathrm{C}$ (aus $\varkappa$ aktuell und $\mathrm{T}^{\circ}$ berechnete $\varkappa_{20}$ ) proportional zum Gehalt an gelösten Salzen sei und dass

c) die angenommene Mischung vollständig sei.

$$
\begin{aligned}
& \mathrm{T}_{\text {Mischwasser }}=\left(\mathrm{T}_{\text {Abwasser }}+a \cdot \mathrm{T}_{\text {Seewasser }}\right) /(1+a) \\
& \varkappa_{\text {Mischwasser }}=\left(\varkappa_{\text {Abwasser }}+a \cdot \varkappa_{\text {Seewasser }}\right) /(1+a)
\end{aligned}
$$

1) Die Technologie der Vermischung ist Gegenstand einer besonderen Studie und wird hier nicht behandelt. 
wobei $a=$ Anteile Seewasser

$\mathrm{T}=$ Temperatur $\left({ }^{\circ} \mathrm{C}\right)$

$\varkappa=$ Leitfähigkeit bei $20^{\circ} \mathrm{C}$

$T_{M i}$ und $\varkappa_{M i}$ erlauben die Berechnung der Dichte des Mischwassers (gemäss S. 350). Dieser Dichtewert liefert im betreffenden Dichtediagramm die in den Tabellen 7-13 angegebene theoretische Einschichtungstiefe.

Dieses Verfahren wurde bei unseren Beobachtungen 1, 3 und 4 in der Hergiswiler Bucht angewendet und die theoretische Einschichtungstiefe für Mischungsverhältnisse von $1+0$ bis $1+10(1$ Teil Abwasser $+a$ Teile Seewasser $)$ berechnet bei Einleitung in 8, 9 und $10 \mathrm{~m}$ Tiefe. Die Dichte des Abwassers wurde aus den entsprechenden Beobachtungen übernommen. Es handelt sich um Situationen, in denen das Abwasser weniger dicht ist als das Seewasser in der Einleitungstiefe und demzufolge aufsteigt (Tab. 6a-c).

Tabelle 6a. Versuch 1 (29. August 1973), Hergiswil. Theoretische Einschichtungstiefe bei Mischung des Abwassers mit a Teilen Seewasser. Abwassertemperatur: $17,25^{\circ} \mathrm{C}$.

Table 6a. Trial 1 (29 August 1973), Hergiswi. Theoretical final stratification depth after mixing wastewater with $a$ parts of lake water. Temperature of wastewater: $17.25^{\circ} \mathrm{C}$.

\begin{tabular}{|c|c|c|c|c|c|c|c|c|c|c|c|}
\hline \multirow{2}{*}{$\begin{array}{l}\text { Einleitungstiefe } \\
\mathrm{m}\end{array}$} & \multicolumn{11}{|c|}{$a$ (Anteile Seewasser) } \\
\hline & 0 & 1 & 2 & 3 & 4 & 5 & 6 & 7 & 8 & 9 & 10 \\
\hline 8 & 4,05 & 5,1 & 5,3 & 6,4 & 6,8 & 7,3 & 7,4 & 7,7 & & & 7,9 \\
\hline 9 & 4,05 & 5,5 & 6,5 & 7,3 & 7,7 & 8,0 & 8,2 & & & & \\
\hline \multirow[t]{2}{*}{10} & 4,05 & 5,8 & 7,5 & 8,0 & 8,3 & 8,4 & 8,6 & 8,9 & 9,0 & & 9,5 \\
\hline & \multicolumn{11}{|c|}{ (Einschichtungstiefe in $\mathrm{m}$ ) } \\
\hline
\end{tabular}

Tabelle $6 \mathrm{~b}$. Versuch 3 (26. September 1973). Abwassertemperatur: $17,5^{\circ} \mathrm{C}$.

Table $6 \mathrm{~b}$. Trial 3 (26 September 1973 ). Temperature of wastewater: $17.5^{\circ} \mathrm{C}$.

\begin{tabular}{|c|c|c|c|c|c|c|c|c|c|c|c|}
\hline \multirow{2}{*}{$\begin{array}{l}\text { Einleitungstiefe } \\
\text { m }\end{array}$} & \multicolumn{11}{|c|}{$a$ (Anteile Seewasser) } \\
\hline & 0 & 1 & 2 & 3 & 4 & 5 & 6 & 7 & 8 & 9 & 10 \\
\hline 8 & 4,7 & 5,0 & 5,9 & 6,5 & 7,0 & 7,15 & 7,25 & 7,31 & 7,4 & 7,5 & 7,55 \\
\hline 9 & 4,7 & 5,3 & 6,5 & 7,3 & 7,55 & 7,7 & 7,8 & 8,1 & 8,15 & 8,2 & 8,22 \\
\hline \multirow[t]{2}{*}{10} & 4,7 & 5,6 & 7,1 & 7,55 & 7,8 & 7,9 & 8,4 & 8,6 & 9,05 & $9,1.2$ & 9,2 \\
\hline & \multicolumn{11}{|c|}{ (Einschichtungstiefe in $\mathrm{m}$ ) } \\
\hline
\end{tabular}

Tabelle 6c. Versuch 4 (31. Juli 1974). Abwassertemperatur: $17,9^{\circ} \mathrm{C}$.

Table $6 \mathrm{c}$. Trial 4 (31 July 1974). Temperature of wastewater: $17.9^{\circ} \mathrm{C}$.

\begin{tabular}{|c|c|c|c|c|c|c|c|c|c|c|c|}
\hline \multirow{2}{*}{$\begin{array}{l}\text { Einleitungstiefe } \\
\mathrm{m}\end{array}$} & \multicolumn{11}{|c|}{$a$ (Anteile Seewasser) } \\
\hline & 0 & 1 & 2 & 3 & 4 & 5 & 6 & 7 & 8 & 9 & 10 \\
\hline 9 & 2,6 & 4,9 & 6,0 & 6,5 & 7,0 & 7,4 & 7,6 & 7,8 & 8,05 & 8,13 & 8,21 \\
\hline \multirow[t]{2}{*}{10} & 2,6 & 5,1 & 6,2 & 7,0 & 7,55 & 7,95 & 8,3 & 8,45 & 8,65 & 8,75 & 8,8 \\
\hline & \multicolumn{11}{|c|}{ (Einschichtungstiefe in $\mathrm{m}$ ) } \\
\hline
\end{tabular}


Tabellen $6 \mathrm{a}-\mathrm{c}$ ergeben erwartungsgemäss, dass die Dichteänderung durch Vermischung mit zunehmendem Anteil Seewasser $(=a)$ einem Endwert zustrebt bzw. dass der relative Effekt am Anfang der Skala am grössten ist. Sie zeigen, dass bei sehr strengen Anforderungen an die erlaubte Abweichung von der theoretischen Einschichtungstiefe ein relativ grosser Anteil von Seewasser erforderlich wäre. So würde eine erlaubte Verlagerung von 10 auf maximal $9 \mathrm{~m}$ in den Versuchen 1 und 3 (6a, $6 \mathrm{~b})$ ein $a$ von 8 , in Versuch $4(6 \mathrm{c})$ von $>10$ erfordern, eine solche von 10 auf $8 \mathrm{~m}$ ein $a$ von $3(6 \mathrm{a})$ bzw. $5(6 \mathrm{~b}, 6 \mathrm{c})$, vollständige Mischung vorausgesetzt.

Diese theoretischen Tiefen weichen von den tatsächlich festgestellten Einschichtungstiefen (Versuch 1:5 m; Versuch 3:7,5 m; Versuch 4: 4 m) sehr stark ab. Als Ursache ist nur eine sehr geringe (ungenügende) Vermischung denkbar.

\subsection{Das notwendige Mischungsverhältnis}

Die in jedem einzelnen Fall zu fordernde Einschichtungstiefe ergibt sich aus limnologischen Gegebenheiten und Erwägungen (Einschichtung unterhalb des photosynthetisch aktiven bzw. produktiven Tiefenbereichs und oberhalb des tieferen Hypolimnions) und praktischen bzw. nutzungsorientierten Bedingungen (ausserhalb des Fassungsbereichs von Trinkwasserwerken, ausserhalb der Badetiefe). Angesichts der individuellen Unterschiede im Schichtungsverhalten der einzelnen Seen ist es wenig sinnvoll, eine einheitliche Einschichtungstiefe anzugeben; sie muss für jeden Fall gesondert gemessen und berechnet werden [5]. Aus dem gleichen Grund ist es schwierig, einen Toleranzbereich der Mischungsverhältnisse anzugeben, zumal der Dichtegradient im Laufe des Jahres jeweils von Null bis zum Maximalwert der Hauptstagnation ansteigt und durch die Zirkulation wieder abgebaut wird.

Immerhin soll im folgenden versucht werden, anhand realistischer Voraussetzungen einen Überblick über die Verhältnisse zu gewinnen, wie sie im konkreten Fall auftreten können, und daraus eine Richtlinie über das bei einer Projektierung vorzusehende Mischungsverhältnis abzuleiten.

Ohne besondere Vorkehren mischt sich das Abwasser, wie festgestellt, beim heutigen Modus der Einleitung ungenügend mit dem Seewasser. Die Häufigkeitsverteilung der daran beteiligten Anteile Seewasser mit dem Abwasser entspricht einer angesteckten kontinuierlichen Verteilung, bei der der häufigste und der Medianwert zwischen $0+1$ und $1+1$ liegen (Abb. 10).

Um das Abwasser in einer vertretbaren Tiefe einzuschichten, ist gemäss Tabellen $6 a-c$ ein Mischungsverhältnis von etwa $1+5$ notwendig. Dieses wird im folgenden als Arbeitshypothese verwendet.

Bei ungenügender Mischung besteht die Gefahr, dass ein Teil des Abwassers, nämlich der mit weniger als 5 Teilen Seewasser vermischte Anteil, höher steigt als $2 \mathrm{~m}$. Wir stellen deshalb die allgemeine Forderung, dass beim Mischungsvorgang, egal, in welcher Weise er schliesslich realisiert wird, nicht mehr als 5\% des Abwassers in einem schlechteren Verhältnis als $1+4$ gemischt werden bzw. dass $95 \%$ des Abwassers $(=1$ Teil) tatsächlich mit 5 Teilen Seewasser eingemischt werden müssen (Abb. 11).

Ein Mischungsverhältnis von $(1+>6)$ bringt praktisch keinen weiteren Nutzeffekt, während der Unterschied zwischen $(1+3)$ und $(1+4)$ bezüglich Effekt beträchtlich ist und die Einschichtungstiefe stark beeinflusst. Das Mischverhältnis 


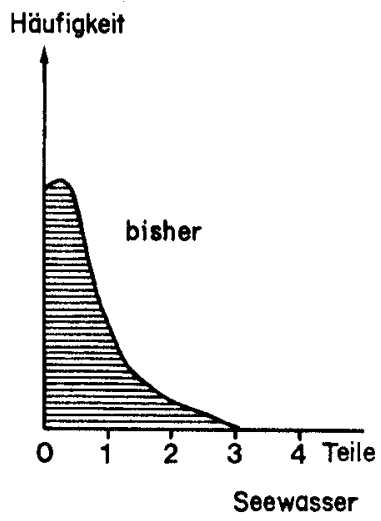

Abb. 10. Verteilung der Häufigkeit der natürlichen Mischungsfraktionen.

Fig. 10. Frequency distribution of normal mixing ratios.

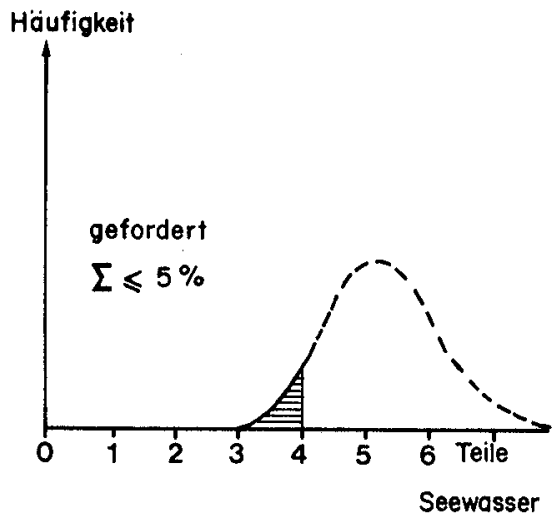

Abb. 11. Geforderte Häufigkeitsverteilung der Mischungsfraktionen.

Fig. 11. Required frequency distribution of mixing ratios.

von $1+5$ bringt das Abwasser-Seewasser-Gemisch in der grossen Mehrzahl aller Fälle $2 \mathrm{~m}$ über der Einleitungstiefe zur Einschichtung (Wegtransport des Gemisches durch eine Seeströmung vorausgesetzt; siehe S. 366).

Die zu erreichende Tiefe der Einschichtung (nicht der Einleitung!) ermittelt sich auf Grund limnologischer Kriterien nach GÄCHTER [5]. Bei einer Einschichtungstoleranz von $2 \mathrm{~m}$, die während eines grossen Teils der (produktiven!) Sommerstagnationsphase nahezu voll nach oben beansprucht wird (kurze herbstliche Phasen mit umgekehrten Verhältnissen, d.h. Absinken des Gemisches, fallen kaum in Betracht), ist es notwendig, diese in der Berechnung der Einleitungstiefe $z u$ berücksichtigen, d.h. um die endgültige Einleitungstiefe zu erhalten, ist die Einschichtungstiefe nach [5] um $2 m$ zu vergrössern.

Zur Verifizierung werden im folgenden je 3 Messprofile aus dem Bodensee-Untersee ("Rheinsee", Seemitte Höhe Berlingen, Tab. 7-9) und aus dem BodenseeObersee (Seemitte Höhe Hagnau-Münsterlingen, Tab. 10-12) unter Annahme verschiedener Abwassertemperaturen durchgerechnet.

Tabelle 7a. Berlingen (25. Juni 1973). Theoretische Einleitungs- und Einschichtungstiefe. Abwassertemperatur: $17^{\circ} \mathrm{C}$.

Table 7 a. Berlingen (25 June 1973). Theoretical discharge and final stratification depth. Temperaturc of wastewater: $17^{\circ} \mathrm{C}$.

\begin{tabular}{lllllllll}
\hline Einleitungstiefe & \multicolumn{2}{l}{ (Anteile Seewasser) } & & & & \\
$\mathrm{m}$ & 0 & 1 & 2 & 3 & 4 & 5 & 6 & 7 \\
\hline 14 & 12,7 & 13,5 & 13,6 & 13,7 & 13,8 & 13,9 & 13,9 \\
16 & 12,7 & 14,1 & 14,8 & 15,1 & 15,3 & 15,4 & $\mathbf{1 5 , 5}$ \\
& (Einschichtungstiefe in m) \\
\hline
\end{tabular}


Tabelle $7 \mathrm{~b}$. Berlingen (25. Juni 1973). Abwassertemperatur: $15^{\circ} \mathrm{C}$.

Table $7 \mathrm{~b}$. Berlingen (25 June 1973). Temperature of wastewater: $15^{\circ} \mathrm{C}$.

\begin{tabular}{|c|c|c|c|c|c|c|c|c|}
\hline \multirow{2}{*}{$\begin{array}{l}\text { Einleitungstiefe } \\
\mathrm{m}\end{array}$} & \multicolumn{8}{|c|}{$a$ (Anteile Seewasser) } \\
\hline & 0 & 1 & 2 & 3 & 4 & 5 & 6 & 7 \\
\hline 11 & 10,1 & 10,3 & 10,7 & 10,8 & 10,8 & 10,8 & 10,9 & 10,9 \\
\hline 13 & 10,1 & 11,4 & 12,1 & 12,2 & 12,4 & 12,6 & 12,6 & 12,7 \\
\hline 14 & 10,1 & 12,1 & 12,6 & 13,1 & 13,3 & 13,4 & 13,6 & 13,7 \\
\hline 15 & 10,1 & 12,5 & 13,2 & 13,6 & 13,8 & 14,0 & 14,1 & 14,2 \\
\hline \multirow[t]{2}{*}{16} & 10,1 & 12,7 & 13,7 & 14,0 & 14,6 & 14,8 & 14,8 & 15,0 \\
\hline & \multicolumn{8}{|c|}{ (Einschichtungstiefe in $\mathrm{m}$ ) } \\
\hline
\end{tabular}

Tabelle 8a. Berlingen (20.August 1973). Theoretische Einleitungs- und Einschichtungstiefe. Abwassertemperatur: $15^{\circ} \mathrm{C}$.

Table 8a. Berlingen (20 August 1973). Theoretical discharge and final stratification depth. Temperature of wastewater: $15^{\circ} \mathrm{C}$.

\begin{tabular}{lllllllll}
\hline Einleitungstiefe & \multicolumn{9}{c}{ (Anteile Seewasser) } & & & & \\
$\mathrm{m}$ & 0 & 1 & 2 & 3 & 4 & 5 & 6 & 7 \\
\hline 13 & 15,9 & 14,3 & 13,8 & 13,6 & 13,5 & 13,4 & 13,4 \\
15 & 15,9 & 15,5 & 15,3 & 15,2 & 15,2 & 15,1 & 15,1 \\
16 & 15,9 & 16,0 & 16 & 16 & 16 & 16 & 16 \\
& & & & & & \\
\end{tabular}

Tabelle $8 \mathrm{~b}$. Berlingen (20. August 1973). Abwassertemperatur: $17^{\circ} \mathrm{C}$.

Table 8 b. Berlingen (20 August 1973). Temperature of wastewater: $17^{\circ} \mathrm{C}$.

\begin{tabular}{lllllllll}
\hline Einleitungstiefe & \multicolumn{9}{l}{ (Anteile Seewasser) } & & & & \\
$\mathrm{m}$ & 0 & 1 & 2 & 3 & 4 & 5 & 6 & 7 \\
\hline 13 & 13,7 & 13,4 & 13,26 & 13,2 & 13,1 & & \\
14 & 13,7 & 13,8 & 13,9 & 13,9 & 13,9 & 13,9 & 13,9 \\
15 & 13,7 & 14,25 & 14,5 & 14,7 & 14,7 & 14,8 & 14,8 \\
16 & 13,7 & 15,0 & 15,4 & 15,6 & 15,6 & 15,7 & 15,75 \\
& & & & & & \\
\hline
\end{tabular}

Tabelle 9a. Berlingen (17. September 1973). Theoretische Einleitungs- und Einschichtungstiefe Abwassertemperatur: $15^{\circ} \mathrm{C}$.

Table 9a. Berlingen (17 September 1973). Theoretical discharge and final stratification depth. Temperature of wastewater: $15^{\circ} \mathrm{C}$.

\begin{tabular}{lllllllll}
\hline Einleitungstiefe & \multicolumn{9}{c}{ (Anteile Seewasser) } & & & & \\
$\mathrm{m}$ & 0 & 1 & 2 & 3 & 4 & 5 & 6 & 7 \\
\hline 12 & 14,0 & 13,0 & 12,6 & 12,5 & 12,3 & 12,3 & 12,25 \\
14 & 14,0 & 14,1 & 14,1 & 14,0 & 14,0 & 14,0 & 14,0 \\
16 & 14,0 & 15,0 & 15,4 & 15,6 & 15,7 & 15,7 & 15,8 \\
& & & & \\
\end{tabular}


Tabelle 9b. Berlingen (17. September 1973). Abwassertemperatur: $17^{\circ} \mathrm{C}$.

Table $9 \mathrm{~b}$. Berlingen (17 September 1973). Temperature of wastewater: $17^{\circ} \mathrm{C}$.

\begin{tabular}{|c|c|c|c|c|c|c|c|c|}
\hline \multirow{2}{*}{$\begin{array}{l}\text { Einleitungstiefe } \\
\mathrm{m}\end{array}$} & \multicolumn{8}{|c|}{$a$ (Anteile Seewasser) } \\
\hline & 0 & 1 & 2 & 3 & 4 & 5 & 6 & 7 \\
\hline 14 & 12,1 & 13,2 & 13,6 & 13,8 & 13,9 & 13,9 & 13,9 & \\
\hline 16 & 12,1 & 13,5 & 13,9 & 14,0 & 14,4 & 14,6 & 14,9 & 15,1 \\
\hline 17 & 12,1 & 14,6 & 15,4 & 16,1 & 16,3 & 16,5 & 16,8 & 16,8 \\
\hline \multirow[t]{2}{*}{18} & 12,1 & 16,8 & 17,8 & 17,8 & 17,8 & 17.9 & & \\
\hline & \multicolumn{8}{|c|}{ (Einschichtungstiefe in $\mathrm{m}$ ) } \\
\hline
\end{tabular}

Es ist zu berücksichtigen, dass der Untersee infolge seiner extrem starken Durchströmung eine ungewöhnlich ausgeglichene Temperaturschichtung aufweist, welche eine gleichmässigere und vor allem kleinere Aufstiegshöhe zur Folge hat. Das Beispiel darf nicht verallgemeinert werden; es zeigt hingegen, dass es unerlässlich ist, die Einleitungsfrage seeindividuell zu behandeln.

Bei diesen Beispielen war am 13. August und 10. September 1974 (Tab. 11, 12) für einen maximal tolerablen Aufstieg um $2 \mathrm{~m}$ meist ein Mischungsverhältnis von $1+5$ erforderlich (Minimalwert $1+1$, Maximalwert $1+6$, beide selten). Für den 14. Mai 1974 wäre bei einer Einleitungstiefe von $20 \mathrm{~m}$, was etwa den limnologischen Forderungen [5] entspricht, für $2 \mathrm{~m}$ Aufstiegshöhe ein Verhältnis von $1+7$ erforderlich (Aufstieg bei $1+5$ von 20 auf 13,7 m). Mit einer Abwassertemperatur von $17^{\circ} \mathrm{C}$ wurde allerdings bewusst eine ungünstige Annahme getroffen.

Tabelle 10. Hagnau-Münsterlingen (14. Mai 1974). Theoretische Einleitungs- und Einschichtungstiefe. Abwassertemperatur: $17^{\circ} \mathrm{C}$.

Table 10. Hagnau-Münsterlingen (14 May 1974). Theoretical discharge and final stratification depth. Temperature of wastewater: $17^{\circ} \mathrm{C}$.

\begin{tabular}{|c|c|c|c|c|c|c|c|c|}
\hline \multirow{2}{*}{$\begin{array}{l}\text { Einleitungstiefe } \\
\mathrm{m}\end{array}$} & \multicolumn{8}{|c|}{$a$ (Anteile Seewasser) } \\
\hline & 0 & 1 & 2 & 3 & 4 & 5 & 6 & 7 \\
\hline 16 & 0 & 0 & 4,5 & 5,4 & 5,7 & 5,9 & 6,5 & 7,6 \\
\hline 18 & 0 & 0 & 5,0 & 5,6 & 5,9 & 6,7 & 8,1 & 9,2 \\
\hline \multirow[t]{2}{*}{20} & 0 & 0 & 5,6 & 7,8 & 10,9 & 13,7 & 16,7 & 18,1 \\
\hline & \multicolumn{8}{|c|}{ (Einschichtungstiefe in $\mathrm{m}$ ) } \\
\hline
\end{tabular}

Tabelle 11. Hagnau-Münsterlingen (13. August 1974). Abwassertemperatur: $17^{\circ} \mathrm{C}$. Table 11. Hagnau-Münsterlingen (13 August 1974). Temperature of wastewater: $17^{\circ} \mathrm{C}$.

\begin{tabular}{lllllllll}
\hline Einleitungstiefe & \multicolumn{9}{l}{ (Anteile Seewasser) } & & & \\
$\mathrm{m}$ & 0 & 1 & 2 & 3 & 4 & 5 & 6 & 7 \\
\hline 16 & 14,3 & 14,7 & 14,8 & 14,9 & 14,9 & 14,9 & \\
18 & 14,3 & 15,0 & 15,9 & 17,2 & 17,4 & & \\
20 & 14,3 & 18,0 & 18,6 & 18,9 & 19,0 & & \\
& (Einschichtungstiefe in m) \\
\hline
\end{tabular}


Tabelle 12a. Hagnau-Münsterlingen (10. September 1974). Theoretische Einleitungs- und Einschichtungstiefe. Abwassertemperatur: $17^{\circ} \mathrm{C}$.

Table 12a. Hagnau-Münsterlingen (10 September 1974). Theoretical discharge and final stratification depth. Temperature of wastewater: $17^{\circ} \mathrm{C}$.

\begin{tabular}{llllllllll}
\hline Einleitungstiefe & \multicolumn{7}{c}{ (Anteile Seewasser) } \\
$\mathrm{m}$ & 0 & 1 & 2 & 3 & 4 & 5 & 6 & 7 & 8 \\
\hline 16 & 8,5 & 12,9 & 13,9 & 14,5 & 14,8 & 15,0 & 15,1 & 15,15 & 15,2 \\
18 & 8,5 & 13,2 & 14,9 & 15,5 & 15,8 & 16,0 & 16,15 & $\mathbf{1 6 , 2}$ & $\mathbf{1 6 , 2 5}$ \\
20 & 8,5 & 13,8 & 15,7 & 16,6 & 17,1 & 17,8 & 18,1 & 18,2 & $\mathbf{1 8 , 3}$ \\
& & & & & & & \\
\hline
\end{tabular}

Tabelle $12 \mathrm{~b}$. Hagnau-Münsterlingen (10. September 1974). Abwassertemperatur: $16^{\circ} \mathrm{C}$.

Table $12 \mathrm{~b}$. Hagnau-Münsterlingen (10 September 1974). Temperature of wastewater: $16^{\circ} \mathrm{C}$.

\begin{tabular}{llllllllll}
\hline Einleitungstiefe & \multicolumn{2}{c}{ (Anteile Seewasser) } \\
$\mathrm{m}$ & 0 & 1 & 2 & 3 & 4 & 5 & 6 & 7 & 8 \\
\hline 16 & 9,9 & 13,5 & 14,2 & 14,8 & 15,1 & 15,4 & 15,5 & 15,5 & 15,6 \\
18 & 9.9 & 13,95 & 15,3 & 15,7 & 16,1 & 16,5 & 16,8 & 16,9 & 16,9 \\
20 & 9,9 & 14,8 & 16,2 & 17,0 & 17,6 & 18,05 & 18,2 & 18,3 & 18,4 \\
& & & & & & & & \\
\hline
\end{tabular}

Tabelle 12 c. Hagnau-Münsterlingen (10. September 1974). Abwassertemperatur: $14^{\circ} \mathrm{C}$.

Table 12c. Hagnau-Münsterlingen (10 September 1974). Temperature of wastewater: $14^{\circ} \mathrm{C}$.

\begin{tabular}{|c|c|c|c|c|c|c|c|c|}
\hline \multirow{2}{*}{$\begin{array}{l}\text { Einleitungstiefe } \\
\mathrm{m}\end{array}$} & \multicolumn{8}{|c|}{$a$ (Anteile Seewasser) } \\
\hline & 0 & 1 & 2 & 3 & 4 & 5 & 6 & 7 \\
\hline 16 & 13,5 & 14,9 & 15,4 & 15,6 & 15,7 & 15,8 & 15,8 & \\
\hline \multirow[t]{2}{*}{20} & 13,5 & 16,1 & 17,2 & 17,5 & 18,1 & 18,2 & 18,3 & \\
\hline & \multicolumn{8}{|c|}{ (Einschichtungstiefe in $\mathrm{m}$ ) } \\
\hline
\end{tabular}

Anderseits ist es gemäss den Beispielen Tabellen 7-12 durchaus möglich, dass der tolerable Aufstiegsweg von $2 \mathrm{~m}$ auch durch ein kleineres Mischungsverhältnis erreicht werden kann, wenn besondere Verhältnisse vorliegen. Diese zu quantifizieren erfordert jedoch eine Untersuchung des Sees, welche (in monatlichen Intervallen) mindestens von März bis Oktober eines Jahres andauern und die Aufnahme lückenloser Temperatur- und Leitfähigkeits-Vertikalprofile (1-m-Schritte) sowie Sichttiefenmessungen umfassen muss und durch Messungen der Lichtextinktion im Vertikalschnitt ergänzt sein sollte.

Anderseits darf unseren Beispielen entnommen werden, dass ein Mischungsverhältnis von $1+5$ mit seltenen Ausnahmen normalerweise ausreicht, um den Aufstieg des Mischwassers (in Herbst- und Wintersituationen auch Abstieg) auf $2 \mathrm{~m}$ zu begrenzen.

Aus diesen Gründen erachten wir das Verhältnis von $1+5$ als vertretbaren, allgemeingültigen Richtwert (mit der Möglichkeit einer durch Untersuchungen begründeten Reduktion). 


\section{Die Frage des Wegtransportes des eingeleiteten Abwassers}

Die Hergiswiler Beobachtungen haben als zweites wichtiges Faktum gezeigt, dass bei Vorherrschen einer Seeströmung das eingeleitete Abwasser in Form einer streng zusammenbleibenden Fahne weggetragen wird, bei der offenbar einzelne Wirbelzöpfe Ausnahmen bleiben. Dieser Vorgang ist vergleichbar mit der Bildung einer Rauchfahne eines Feuers bei starkem Wind.

Es macht den Anschein, als ob sich die einzelnen Wirbelzöpfe gegeneinander und gegenüber dem See wie schwermischbare Kompartimente verhalten. Fehlt die Seeströmung (von ScHRöDER [4] als «Restströmung" bezeichnet), so kann sich das eingeleitete Abwasser um die Einleitungstiefe herum in einer sich rasch aufsummierenden Wolke oder Blase akkumulieren, deren Oberkante bis in die Badetiefe hinauf reichen kann. Dies ungeachtet, ob zwischen dem Abwasser bzw. dessen Mischung und dem übrigen See eine Dichtedifferenz besteht oder nicht. Es handelt sich hier um eine sehr folgenschwere Feststellung, der bei der Planung von Kläranlagen grösste Beachtung geschenkt werden muss. Jedenfalls ist es unerlässlich, dass die künftige Einleitungsstelle auf Grund sorgfältiger und bei allen wesentlichen Wetterlagen wiederholter Strömungsmessungen in allen in Frage kommenden Wassertiefen festgelegt wird. Die bisherigen limnologischen Erfahrungen machen es wahrscheinlich, dass eine zuverlässige Strömung gefunden werden kann.

\section{Schlussbemerkungen}

Die vorliegende Arbeit beantwortet die Frage, nach welchem optimalen Modus gereinigtes Abwasser im See untergebracht werden kann (bzw. muss). Sie nimmt aber keine Stellung zur Frage des möglichen oder verantwortbaren Ausmasses einer solchen Einleitung. Mit der gezielten Einschichtung von gereinigtem Abwasser wird vermieden, dass dieses seine trotz Reinigung noch verbliebene Restdüngekapazität zur unmittelbaren Wirkung (= "Dauerfütterung" des Sees) bringen kann. Die nächstfolgende Zirkulation wird das eingeschichtete Abwasser einmischen und alljährlich eine neue Ausgangslage schaffen, so lange bis in der Jahressumme zwischen der sommerlichen hypolimnischen Akkumulation, dem winterlichen (zirkulationsbedingten) Wegtransport via Abfluss und den laufenden Zufuhren durch Oberflächengewässer und gezielte Einleitung ein Dauerzustand (bzw. Steady state) erreicht ist. Dabei drängt sich die Frage auf, welches Volumen Seewasser für die Aufnahme von eingeleitetem Abwasser während einer Stagnationsperiode überhaupt beansprucht werden darf.

Mengenmässig geringfügige Einleitungen werden - bei allem Unbehagen gegenüber jeder Seedüngung - kaum Probleme aufwerfen. Im Falle mengenmässig bedeutender Einleitungen ist es eine Frage der Relation zwischen Abwassermenge bzw. Saisonsumme und dem Volumen der für die Einschichtung zur Verfügung stehenden Schicht des Sees, ob letztere (im einfachsten Fall) für einen einmaligen Durchsatz pro Saison ausreicht, oder ob infolge eines mehrmaligen Durchsatzes eine Aufstockung und dadurch eine entsprechende Erhöhung der Nährstoffgehalte (plus 
weiterer Abwasserkomponenten) erfolgt. Die Forderung jedenfalls, das eingeleitete Abwasser dem Photosyntheseprozess fernzuhalten, wird dadurch höchstens verschärft, und verschärft wird auch die Forderung, dass die Einschichtungstiefe nicht verringert werden darf.

Wir sind uns bewusst, dass diese Massnahme den Eutrophierungsprozess nicht an der Wurzel beeinflusst, zumal die Nährstoffzufuhr zum See als Ganzes unbeeinflusst bleibt; beeinflusst wird lediglich ihre unmittelbare Wirkung auf den (für den See allerdings fundamental wichtigen) Produktionsprozess.

Die Verfasser sind Herrn $\mathrm{Hj}$. Schälchli und Herrn E. Schwager (Werkstätte der Versuchsanlage Tüffenwies der EAWAG) für die rasche Anfertigung der Fernsteuerung dankbar. Dank der Bereitwilligkeit von Herrn Klärmeister A. Keiser, ARA Hergiswil, konnte die Tracerdosierung jeweils reibungslos installiert und betrieben werden. Das Navigationsproblem erforderte eine besonders einfühlende Führung des Motorbootes, wofür Herrn A. Mareš bestens gedankt sei.

\section{Zusammenfassung}

1. In vier Versuchen wurde der Weg von eingeleitetem Abwasser im Seewasserkörper in situ verfolgt (Vierwaldstättersee, Abwasser der Kläranlage Hergiswil). Das Abwasser wurde mit Aluminiumpulver markiert; die Fahne konnte im See mit Sonar (Echograph) registriert werden.

2. Die Messungen im See wurden im Sommer und Herbst 1973 und im Frühjahr und Sommer 1974 durchgeführt. Eine Messung fiel in die beginnende Zirkulationsphase, die übrigen liegen in der Stagnationsphase.

3. Der Austritt des Abwassers erfolgte unter Reynoldszahlen von 1,3 $\cdot 10^{5}$ bis rund $1,9 \cdot 10^{5}$, somit turbulent. Trotzdem war eine ins Gewicht fallende Vermischung des Abwassers mit Seewasser nicht feststellbar. Das Abwasser bleibt als schlecht mischbares Kompartiment beisammen und schichtet sich rasch in jener Seetiefe ein, die die gleiche Dichte aufweist.

4. Herrscht eine Seeströmung, so wird das Abwasser als Fahne weggetragen. Fehlt diese, so wird es in Form einer Blase um die Rohrmündung akkumuliert und kann dadurch bis in die Badetiefe hinauf gelangen.

5. Um eine Akkumulation von Abwasser im See auszuschliessen, wird empfohlen, in jedem einzelnen Fall die Strömungsverhältnisse abzuklären. Eine Einleitung soll nur dort erfolgen, wo eine zuverlässige Seeströmung vorhanden ist.

6. Um eine Einschichtung des Abwassers auf einer vorgewählten Tiefe sicherzustellen, muss die Dichte des Abwassers derjenigen dieser Tiefe angeglichen werden. Als realistisches Mittel ist eine turbulente Vermischung im See (z.B. durch hohe Austrittsgeschwindigkeit des Abwassers erzeugt) denkbar. Die Technik der Vermischung ist aber nicht Gegenstand dieser Arbeit.

7. Vergleiche mit gemessenen Situationen aus dem Bodensee ergeben, dass eine Vermischung von 1 Teil Abwasser mit 5 Teilen Seewasser in der Grosszahl aller Fälle ausreicht, um eine Vertikalbewegung des Gemisches auf maximal $2 \mathrm{~m}$ zu beschränken. In Sonderfällen kann das Verhältnis noch kleiner sein. Das Mischungsverhältnis von $1+5$ wird als allgemeine Richtlinie empfohlen. 
8. Die Studie bezweckt, die (oft unvermeidliche) Einleitung von gereinigtem Abwasser in einen See so zu optimieren, dass die (ebenfalls unvermeidlichen) Schäden möglichst niedrig bleiben. Sie befasst sich nicht mit übergeordneten Gewässerschutzfragen.

\section{SUMMARY}

\section{Discharge of Treated Wastewater in Lakes}

1. The paths of wastewater in a lake were traced four times (Lake of Lucerne, sewage treatment plant Hergiswil). The wastewater was marked with aluminium powder and the cloud in the lake registered by an echo-sounder.

2. Measurements in the lake were made in summer and autumn 1973 and in spring and summer 1974. One measurement was taken at the beginning of the overturn period, the rest in the stagnation period.

3. Concluding from Reynold's figures: $1.3 \cdot 10^{5}$ to about $1.9 \cdot 10^{5}$, wastewater discharge was assumed to be turbulent. In spite of this, mixing with lake water was not established. The wastewater keeps together as a bad mixing compartment and flows to the depth of the same density.

4. If there is a steady current in the lake, the wastewater drifts away, in the form of a plume. If the lake is calm, it accumulates as a cloud at the pipe's mouth and can well up to the depth of bathing.

5. To avoid an accumulation of wastewater in the lake in each single case the currents have to be known. A discharge site should only be placed where the current is steady.

6 . To be sure to have the wastewater at a predicted depth it must have the same density as the water of the required depth. A realistic means to reach this is to have turbulent mixing (e.g. by means of high velocity of the wastewater at the point of discharge) in the lake.

7. Comparisons with events measured in the Lake of Constance showed that mixing one part of wastewater with five parts of lake water is usually enough to limit the vertical movement to 2 meters. In special cases the ratio may be smaller. A general mixing ratio of 1 to 5 is recommended.

8. This study intends only to optimize the (often inevitable) wastewater discharge in a lake, so that the bad effects (also inevitable) are minimized. It does not consider any other aspects of lake restoration.

\section{RÉSUMÉ}

L'intromission de l'eau usée épurée dans un lac

1. Le trajet de l'eau épurée, introduite dans la masse d'eau du lac, a été suivi, in situ, en 4 expériences (lac des Quatre-Cantons; eau épurée de la station d'épuration d'Hergiswil). L'eau épurée a été marquée avec de la poudre d'aluminium; sa trace a pu être enregistrée dans le lac par sonar (échographe).

2. Les mesures ont été poursuivies pendant l'été et l'automne 1973, puis durant le printemps et l'été 1974 . Une mesure a été faite au début de la phase de circulation et les autres pendant la phase de stagnation.

3. La sortie d'eau épurée est considérée comme turbulente avec des chiffres de Reynold entre $1,3 \cdot 10^{5}$ et approx. $1,9 \cdot 10^{5}$. Malgré tout, un mélange des eaux épurées avec l'eau du lac n'était pas perceptible. L'eau épurée reste en bloc, celui-ci se mélange mal et s'insère rapidement dans la couche du lac qui presente la même densité que lui.

4. En présence d'un courant, l'eau épurée est entraînée. S'il n'y a pas de courant, l'eau épurée est accumulée sous forme de poche à l'embouchure de la canalisation et peut, par conséquent, se monter jusqu'au niveau auquel on se baigne. 
5. Pour qu'une accumulation de l'eau épurée clans le lac soit exclue, on recommande d'étudier les courants pour chaque cas particulier. Une intromission doit avoir lieu seulement là où il y a un courant certain.

6. Pour que les eaux épurées soient vraiement introduites à la profondeur choisie, la densité des eaux épurées doit être adaptée à celle qui existe à cette profondeur. Comme moyen pratique, on envisage une mixtion turbulente dans le lac (p. ex. en projetant l'eau épurée avec force dans le lac). La technique du mélange n'est cependant pas le but de ce travail.

7. Comparé avec les données provenant du lac de Constance, il s'avère qu'un mélange d'un volume d'eau épurée avec cinq volumes d'eau du lac suffit, dans la plupart des cas, pour qu'un mouvement vertical du mélange se limite au maximum à $2 \mathrm{~m}$. Dans les cas particuliers, la proportion peut être encore plus petite. Comme directives générales, on peut recommander le rapport de mélange $1+5$.

8. Cette étude à pour seul but d'optimiser l'intromission (souvent inévitable) de l'eau usée épurée dans un lac, de façon à ce que les dégâts (également inévitables) restent le plus faible possible. Elle ne s'occupe pas des problèmes de protection des eaux d'un degré supérieur.

\section{LITERATURVERZEICHNIS}

[1] Versuchsanstalt für Wasserbau, Hydrologie und Glaziologie an der ETHZ, Bevicht über Modellversuche zum Problem dev Einleitung von geklärtem Abwasser in Seen (Zürich, Juni 1973).

[2] Eidg. Departement des Innern, Vorläufige Empfehlungen über die regelmässige Untersuchung der schweizerischen Obevflächengewässer (Bern, 29. Mai 1974).

[3] Thomas, E. A., Untersuchungen über Auswirkungen und Folgen der Einschichtung von Abwässern in Seen, Gas, Wasser, Abwasser 49, Nr. 11, 378-384 (1969).

[4] ScHrödER, R., Strömungsverhältnisse im Bodensee-Untersee und der Wasseraustausch zwischen den einzelnen Seebecken, Internationale Gewässerschutzkommission für den Bodensee und Landesstelle für Gewässerkunde und wasserwirtschaftliche Planung BadenWürttemberg 1974.

[5] GÄCHTER, R., Zuv Frage dev Einleitung von geveinigtem Abwasser in Seen, Schweiz. Z. Hydrol. $33,73-84$ (1971).

Adresse der Autoren:

H. Bührer, dipl. rer. nat., EAWAG, CH-8600 Dübendorf; Prof. Dr. H. Ambühl, EAWAG, $\mathrm{CH}-8600$ Dübendorf. 